\title{
Modeling of Concrete Creep Based on Microprestress-solidification Theory
}

\author{
P. Havlásek, M. Jirásek
}

\begin{abstract}
Creep of concrete is strongly affected by the evolution of pore humidity and temperature, which in turn depend on the environmental conditions and on the size and shape of the concrete member. Current codes of practice take that into account only approximately, in a very simplified way. A more realistic description can be achieved by advanced models, such as model B3 and its improved version that uses the concept of microprestress. The value of microprestress is influenced by the evolution of pore humidity and temperature. In this paper, values of parameters used by the microprestress-solidification theory (MPS) are recommended and their influence on the creep compliance function is evaluated and checked against experimental data from the literature. Certain deficiencies of MPS are pointed out, and a modified version of MPS is proposed.
\end{abstract}

Keywords: creep, concrete, compliance function, Kelvin chain, solidification, microprestress, finite elements.

\section{Introduction}

In contrast to metals, concrete exhibits creep already at room temperature. This phenomenon results in a gradual but considerable increase in deformation at sustained loads, and needs to be taken into account in the design and analysis of concrete structures. The present paper examines an advanced concrete creep model, which extends the original B3 model [1] and uses the concepts of solidification $[5,6]$ and microprestress $[3,4,2]$. The main objective of the paper is to clarify the role of non-traditional model parameters and provide hints on their identification. The creep tests performed by Kommendant, Polivka, and Pirtz [8], Nasser and Neville [9], and Fahmi, Polivka and Bresler [7] are used as a source of experimental data, which are compared with the results of numerical simulations. References [8] and [9] were focused mainly on creep of sealed concrete specimens subjected to elevated but constant temperatures. Reference [7] studied creep under variable temperature for both sealed and drying specimens. The same references were used in [2] to demonstrate the functionality of the Microprestress-Solidification Theory, which is the constitutive model described in Section 2. All numerical computations have been performed using the finite element package OOFEM [10-12] developed mainly at the CTU in Prague by Bořek Patzák.

\section{Description of the material model}

The complete constitutive model for creep and shrinkage of concrete can be represented by the rheological scheme shown in Figure 1. It consists of (i) a non-aging elastic spring, representing instantaneous elastic deformation, (ii) a solidifying Kelvin chain, representing short-term creep, (iii) an aging dashpot with viscosity dependent on the microprestress, $S$, representing long-term creep, (iv) a shrinkage unit, representing volume changes due to drying, and (v) a unit representing thermal expansion. All these units are connected in series, and thus the total strain is the sum of the individual contributions, while the stress transmitted by all units is the same. Attention is focused here on the mechanical strain, composed of the first three contributions to the total strain, which are stress-dependent. In the experiments, shrinkage and thermal strains were measured separately on load-

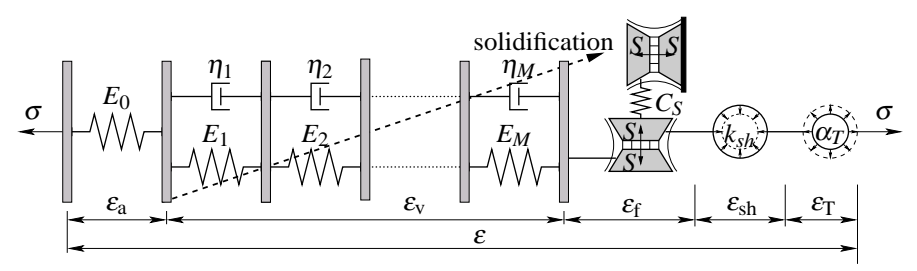

Fig. 1: Rheological scheme of the complete hygro-thermo-mechanical model 
free specimens and subtracted from the strain of the loaded specimen under the same environmental conditions. It should be noted that even after subtraction of shrinkage and thermal strain, the evolution of mechanical strain is affected by humidity and temperature. The reference case is so-called basic creep, i.e. creep in sealed conditions and at room temperature. Dry concrete creeps less than wet concrete, but the process of drying accelerates creep. Elevated temperature leads to faster cement hydration and thus to faster reduction of compliance due to aging, but it also accelerates the viscous processes that are at the origin of creep and the process of microprestress relaxation.

Solidification theory [5] reflects the process of concrete aging due to cement hydration, which leads to the deposition of new layers of solidified hydration products (mainly calcium-silicate-hydrate gels, C-S$\mathrm{H})$. It is assumed that the creep of C-S-H is described by non-aging viscoelasticity, and aging is caused by the growth in volume of the solidified material, which leads to a special structure of the compliance function, reflected by model B3. According to this model, basic creep is described by a compliance function of the form

$$
\begin{aligned}
J_{b}\left(t, t^{\prime}\right)= & q_{1}+q_{2} \int_{t^{\prime}}^{t} \frac{n s^{-m}}{s-t^{\prime}+\left(s-t^{\prime}\right)^{1-n}} \mathrm{~d} s+ \\
& q_{3} \ln \left[1+\left(t-t^{\prime}\right)^{n}\right]+q_{4} \ln \frac{t}{t^{\prime}}, \quad t \geq t^{\prime},
\end{aligned}
$$

where $t$ is the current time (measured as the age of the concrete, expressed in days), $t^{\prime}$ is the age at load application, $n=0.1, m=0.5$, and $q_{1}, q_{2}, q_{3}$ and $q_{4}$ are parameters determined by fitting of experimental results or estimated from concrete composition and strength using empirical formulae. The first (constant) term corresponds to the compliance $q_{1}=1 / E_{0}$ of the elastic spring in Figure 1, the second and third terms to the solidifying viscoelastic material (in numerical simulations approximated by a solidifying Kelvin chain), and the fourth term to an aging viscous dashpot with viscosity $\eta_{f}(t)=t / q_{4}$.

Microprestress-solidification theory is an extension of the above model to variable humidity and temperature. It replaces the explicit dependence of viscosity $\eta_{f}$ on time by its dependence on the socalled microprestress, $S$, which is governed by a separate evolution equation. The microprestress is understood as the stress in the microstructure generated due to large localized volume changes during the hydration process. It builds up at very early stages of microstructure formation and then is gradually reduced by relaxation processes. The microprestress is considered to be much bigger than any stress acting on the macroscopic level, and therefore it is not influenced by the macroscopic stress. Additional microprestress is generated by changes in internal relative humidity and temperature. This is described by the non-linear differential equation

$$
\frac{\mathrm{d} S}{\mathrm{~d} t}+\psi_{S}(T, h) c_{0} S^{2}=k_{1}\left|\frac{\mathrm{d}(T \ln h)}{\mathrm{d} t}\right|
$$

in which $T$ denotes the absolute temperature, $h$ is the relative pore humidity (partial pressure of water vapor divided by the saturation pressure), $c_{0}$ and $k_{1}$ are constant parameters, and $\psi_{S}$ is a variable factor that reflects the acceleration of microprestress relaxation at higher temperature and its deceleration at lower humidity (compared to the standard conditions). Owing to the presence of the absolute value operator on the right-hand side of (2), additional microprestress is generated by both drying and wetting, and by both heating and cooling, as suggested in [2].

The dependence of factor $\psi_{S}$ on temperature and humidity is assumed in the form

$$
\begin{gathered}
\psi_{S}(T, h)=\exp \left[\frac{Q_{S}}{R}\left(\frac{1}{T_{0}}-\frac{1}{T}\right)\right] . \\
{\left[\alpha_{S}+\left(1-\alpha_{S}\right) h^{2}\right]}
\end{gathered}
$$

where $Q_{S}$ is the activation energy, $R$ is the Boltzmann constant, $T_{0}$ is the reference temperature (room temperature) in absolute scale and $\alpha_{S}$ is a parameter. The default parameter values recommended in [2] are $Q_{S} / R=3000 \mathrm{~K}$ and $\alpha_{S} \approx 0.1$.

As discussed in [3], high microprestress facilitates sliding in the microstructure and thus accelerates creep. Therefore, the viscosity of the dashpot that represents long-term viscous flow is assumed to be inversely proportional to the microprestress. This viscosity acts as a proportionality factor between the flow rate and the stress. The model is thus described by the equations

$$
\begin{aligned}
\sigma & =\eta_{f} \frac{\mathrm{d} \varepsilon_{f}}{\mathrm{~d} t} \\
\eta_{f} & =\frac{1}{c S}
\end{aligned}
$$

with a constant parameter $c$, which is not independent and can be linked to the already introduced parameters. It suffices to impose the requirement that, under standard conditions $\left(T=T_{0}\right.$ and $\left.h=1\right)$ and constant stress, the evolution of flow strain should be logarithmic and should exactly correspond to the last term of the compliance function (1) of model B3. A simple comparison reveals that $c=c_{0} q_{4}$. At the same time, we obtain the appropriate initial condition for microprestress, which must supplement differential equation (2). The initial condition reads $S_{0}=1 /\left(c_{0} t_{0}\right)$, where $t_{0}$ is a suitably selected time that precedes the onset of drying and temperature variations.

As already mentioned, parameters $q_{1}, q_{2}, q_{3}$ and $q_{4}$ are related to basic creep and can be predicted 
from the composition of the concrete mixture and its average 28-day compressive strength using empirical formulae [1]. The part of the compliance function that contains $q_{2}$ and $q_{3}$ is related to viscoelastic effects in the solidifying part of the model. In numerical simulations, this part of compliance is approximated by Dirichlet series corresponding to a solidifying Kelvin chain. The stiffnesses and viscosities of individual Kelvin units can be conveniently determined from the continuous retardation spectrum of the non-aging compliance function that describes the solidifying constituent. The flow term representing long-term creep is handled separately. For general applications with variable environmental conditions, it is necessary to determine parameters $c_{0}$ and $k_{1}$ that appear in the microprestress evolution equation (2) and indirectly affect the flow viscosity. The model response is also influenced by parameters $Q_{S} / R$ and $\alpha_{S}$, for which default values have been recommended. As will be shown later, a better agreement with experimental results can be obtained if the default values are adjusted. Also, the assumption that changes of $T \ln h$ contribute to the build-up of microprestress independently of their sign will be shown to be too simplistic.

\section{$3 \quad$ Numerical simulations}

In this section, experimental data are compared to results obtained with MPS theory, which reduces to the standard B3 model in the special case of basic creep. All examples concerning drying and thermally induced creep have been run as a staggered problem, with the heat and moisture transport analyses preceding the mechanical analysis. The available experimental data contained the mechanical strains (due to elasticity and creep), with the thermal and shrinkage strains subtracted.

\subsection{Experiments of Kommendant, Polivka and Pirtz (1976)}

At the time of writing, the original report was not available to the authors of the present paper; therefore the experimental data as well as the recommended basic creep parameters $\left(q_{1}=20.0, q_{2}=70.0\right.$, $q_{3}=5.6$ and $q_{4}=7.0$, all in $10^{-6} / \mathrm{MPa}$ ) were taken from [2]. Under constant uniaxial load and constant temperature, it is assumed that there are similar conditions in the whole specimen. This allowed all computations to be carried out on just one finite element.

Figure 2 shows the experimental (points) and calculated (curves) compliance functions for two different ages at loading and three different levels of temperature. For the younger age at loading, $t^{\prime}=$ 28 days, the computed curves provide an excellent fit of the measured data for temperatures $23^{\circ} \mathrm{C}$ and
$43{ }^{\circ} \mathrm{C}$. For the highest temperature, $T=71^{\circ} \mathrm{C}$, the compliance is somewhat overpredicted for load durations from 1 week to 2 years. For the higher age at loading, $t^{\prime}=90$ days, the measured data are underpredicted for all temperatures, but starting from load durations of 10 days all the creep rates are predicted very well. The reduced accuracy can be attributed to the general tendency of the B3 model to overemphasize the effect of aging.
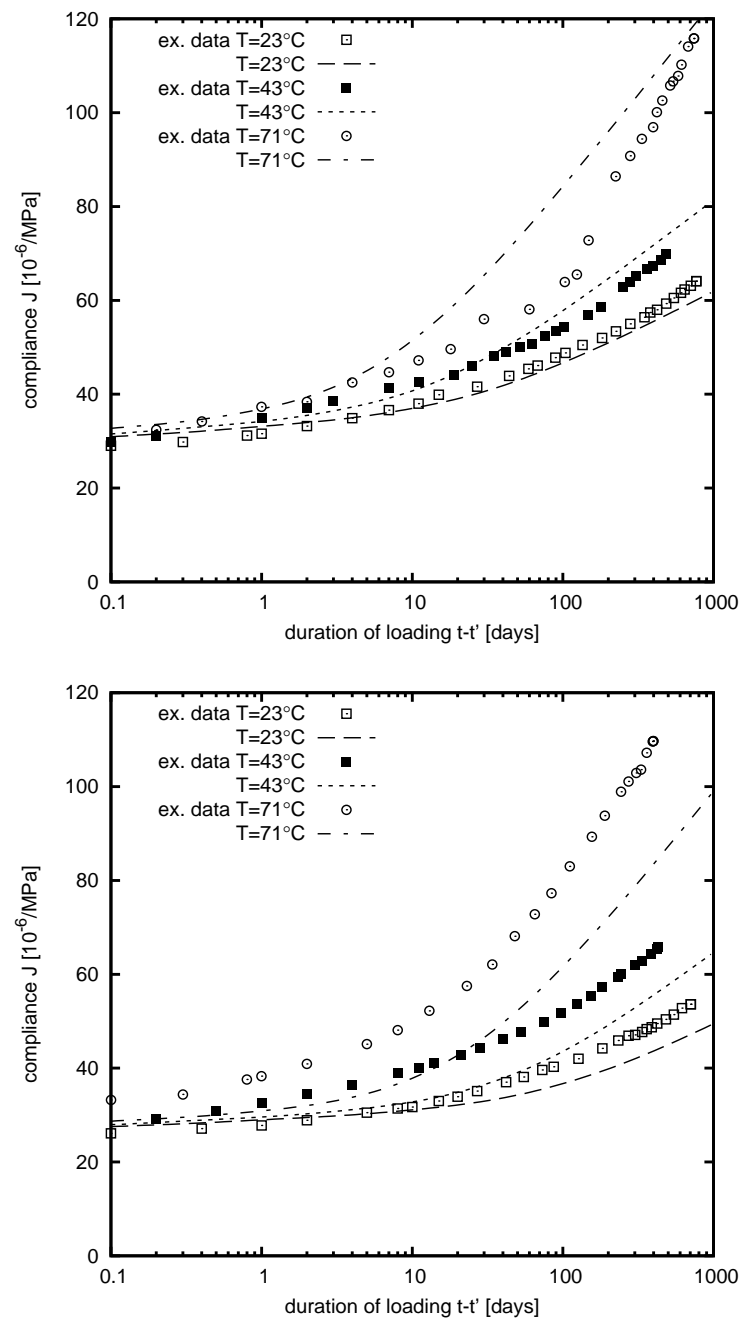

Fig. 2: Experimental data (Kommendant, Polivka and Pirtz) and computed compliance functions for age at loading $t^{\prime}=28$ days (top) and $t^{\prime}=90$ days (bottom)

In this example, the present results agree with those presented in the original work [2], which verifies the correct implementation. The calculated data are independent of parameters $c_{0}$ and $k_{1}$. One can obtain exactly the same curves as $T=23^{\circ} \mathrm{C}$ in Figure 2 just by substituting parameters $q_{1}-q_{4}$, age at loading $t^{\prime}$ and the duration of loading $t-t^{\prime}$ into the full version of the B3 model; see equation 1 .

The original B3 model contains a simple extension to basic creep at constant elevated temperatures; see section 1.7.2 in [1]. The actual age at loading and the load duration are replaced by the equivalent age 
and the equivalent load duration, which evolve faster at elevated temperatures. The calculated compliance functions for default values of activation energies, assumed water content $w=200 \mathrm{~kg} / \mathrm{m}^{3}$ and average 28-day compressive strength $\bar{f}_{c}=35 \mathrm{MPa}$ are shown in Figure 3. For loading at age $t^{\prime}=28$ days, the initial compliance is overestimated and for the highest temperature $71^{\circ} \mathrm{C}$ the rate of creep for longer loading durations is too low. The compliance functions for loading at age $t^{\prime}=90$ days fit the experimental data nicely except for the highest temperature. In all cases the rate of creep is captured better by MPS theory.
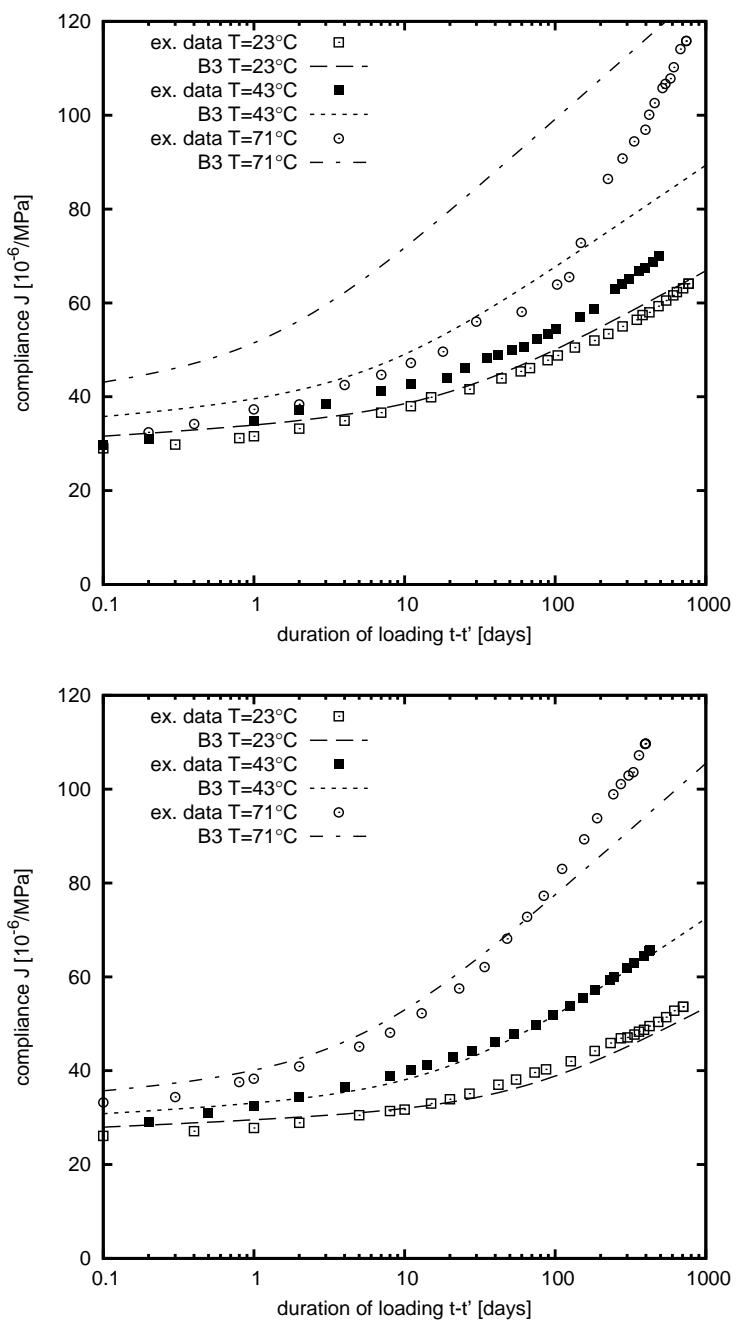

Fig. 3: Experimental data (Kommendant, Polivka and Pirtz) and computed compliance functions for age at loading $t^{\prime}=28$ days (top) and $t^{\prime}=90$ days (bottom) using the original model B3

\section{$3.2 \quad$ Experiments of Nasser and Neville (1965)}

Nasser and Neville studied the creep of cylindrical concrete specimens subjected to three different levels of temperature. In their experiments, all speci- mens were sealed in water-tight jackets and placed in a water bath in order to guarantee constant temperature. At the age of 14 days the specimens were loaded to $35 \%, 60 \%$ or $69 \%$ of the average compressive strength at the time of loading; unfortunately, just the lowest load level is in the range in which concrete creep can be considered as linear. Paper [9] does not contain enough information to allow the parameters of MPS theory to be predicted, but the values $q_{1}=15, q_{2}=80, q_{3}=24$ and $q_{4}=5$ (all in $10^{-6} / \mathrm{MPa}$ ) published in [2] again provide good agreement at room temperature, see the first graph in Figure 4.
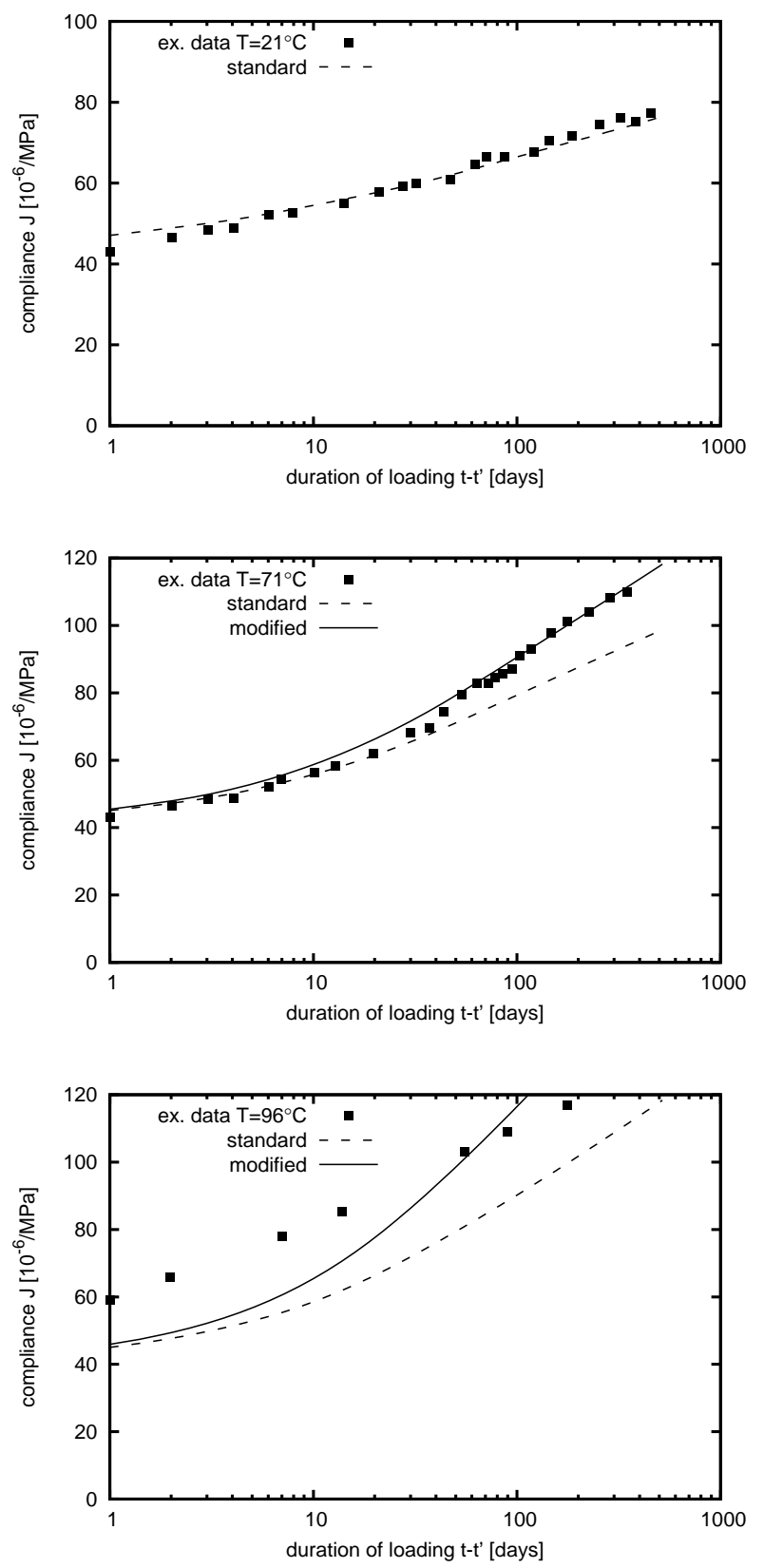

Fig. 4: Experimental data (Nasser and Neville) and compliance functions for temperatures $21^{\circ} \mathrm{C}, 71^{\circ} \mathrm{C}$ and $96^{\circ} \mathrm{C}$ 


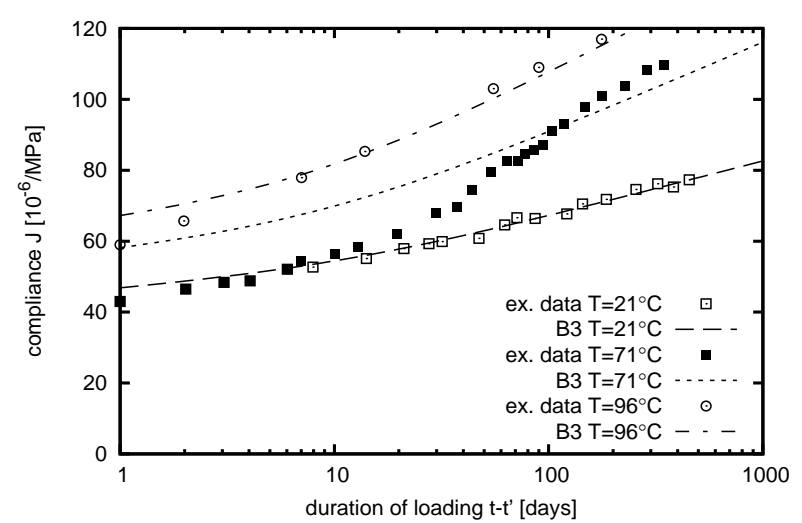

Fig. 5: Experimental data (Nasser and Neville) and compliance functions obtained with the original model B3 for temperatures $21^{\circ} \mathrm{C}, 71^{\circ} \mathrm{C}$ and $96^{\circ} \mathrm{C}$

For the higher temperature, $T=71^{\circ} \mathrm{C}$, the agreement is good up to 20 days at loading, but afterwards the computed rate of creep is too low. A remedy can be sought in modifying the activation energy. Reduction of $Q_{S} / R$ from the default value $3000 \mathrm{~K}$ to the adjusted value of $2200 \mathrm{~K}$ leads to an excellent fit; see the curve labeled in Figure 4 as modified. Unfortunately, the prediction for the highest temperature $\left(T=96{ }^{\circ} \mathrm{C}\right)$ is improved only partially.

Changes in activation energy have no influence on the results when the temperature is close to the room temperature. Before loading, the specimens had been subjected to an environment at the given temperature, which accelerated the hydration processes in concrete, i.e. the maturity of concrete. In other words, the higher the temperature, the lower the initial compliance. On the other hand, for longer periods of loading the higher temperature accelerates the rate of bond breakages, which accelerates creep. This justifies the shape of the obtained curve for the medium temperature, which is different from the one published in [2], where the initial compliance for this temperature was higher than for the room temperature.

The compliance functions obtained with the B3 model are shown for all tested temperatures in Figure 5. Again, default values were used for the activation energies, assumed water content $w=200 \mathrm{~kg} / \mathrm{m}^{3}$ and compressive strength $\bar{f}_{c}=35 \mathrm{MPa}$. Experimental data for the room temperature and for the highest temperature are captured nicely, but the compliance function for $T=71^{\circ} \mathrm{C}$ is overestimated (until 100 days of load duration), and the final rate of creep seems to be too low.

\subsection{Experiments of Fahmi, Polivka and Bresler (1972)}

In these experiments, all specimens had the shape of a hollow cylinder with inner diameter $12.7 \mathrm{~cm}$, outer diameter $15.24 \mathrm{~cm}$ and height $101.6 \mathrm{~cm}$. The weight ratio of the components of the concrete mixture was water : cement: aggregates $=0.58: 1: 2$. From this we can estimate that the concrete mixture contained approximately $520 \mathrm{~kg}$ of cement per cubic meter. The average 21-day compressive strength was 40.3 MPa. Using CEB-FIP recommendations, the 28-day strength can be estimated as $42.2 \mathrm{MPa}$. The experiment was performed for four different histories of loading, temperature and relative humidity. The loading programs of the first two specimens are summarized in Tables 1 and 2, the other two loading programs with cyclic thermal loading are specified in Tables 3 and 4 .

Table 1: Testing program of the sealed specimen with one temperature cycle (Data set \#1)

\begin{tabular}{ccrc}
\hline $\begin{array}{c}\text { time duration } \\
\text { [day }]\end{array}$ & $\begin{array}{c}T \\
{\left[{ }^{\circ} \mathrm{C}\right]}\end{array}$ & $\begin{array}{r}\mathrm{RH} \\
{[\%]}\end{array}$ & $\begin{array}{c}\sigma \\
{[\mathrm{MPa}]}\end{array}$ \\
\hline 21 & 23 & 100 & 0 \\
37 & 23 & 98 & -6.27 \\
26 & 47 & 98 & -6.27 \\
82 & 60 & 98 & -6.27 \\
10 & 23 & 98 & -6.27 \\
25 & 23 & 98 & 0 \\
\hline
\end{tabular}

Table 2: Testing program of the drying specimen with one temperature cycle (Data set \#2)

\begin{tabular}{ccrc}
\hline $\begin{array}{c}\text { time duration } \\
{[\text { day }]}\end{array}$ & $\begin{array}{c}T \\
{\left[{ }^{\circ} \mathrm{C}\right]}\end{array}$ & $\begin{array}{c}\mathrm{RH} \\
{[\%]}\end{array}$ & $\begin{array}{c}\sigma \\
{[\mathrm{MPa}]}\end{array}$ \\
\hline 18 & 23 & 100 & 0 \\
14 & 23 & 50 & 0 \\
37 & 23 & 50 & -6.27 \\
108 & 60 & 50 & -6.27 \\
10 & 23 & 50 & -6.27 \\
25 & 23 & 50 & 0 \\
\hline
\end{tabular}

Table 3: Testing program of the sealed specimen subjected to several temperature cycles (Data set \#3). Asterisks denote a section which is repeated $4 \times$

\begin{tabular}{ccrc}
\hline $\begin{array}{c}\text { time duration } \\
\text { [day }]\end{array}$ & $\begin{array}{c}T \\
{\left[{ }^{\circ} \mathrm{C}\right]}\end{array}$ & $\begin{array}{c}\mathrm{RH} \\
{[\%]}\end{array}$ & $\begin{array}{c}\sigma \\
{[\mathrm{MPa}]}\end{array}$ \\
\hline 21 & 23 & 100 & 0 \\
35 & 23 & 98 & -6.27 \\
9 & 40 & 98 & -6.27 \\
5 & 60 & 98 & -6.27 \\
14 & 23 & 98 & -6.27 \\
$7^{*}$ & 60 & 98 & -6.27 \\
$7^{*}$ & 23 & 98 & -6.27 \\
7 & 60 & 98 & -6.27 \\
12 & 23 & 98 & -6.27 \\
40 & 23 & 98 & 0 \\
\hline
\end{tabular}


Table 4: Testing program of the drying specimen subjected to several temperature cycles (Data set \#4). Asterisks denote a section which is repeated $4 \times$

\begin{tabular}{cccc}
\hline $\begin{array}{c}\text { time duration } \\
\text { [day }]\end{array}$ & $\begin{array}{c}T \\
{\left[{ }^{\circ} \mathrm{C}\right]}\end{array}$ & $\begin{array}{c}\mathrm{RH} \\
{[\%]}\end{array}$ & $\begin{array}{c}\sigma \\
{[\mathrm{MPa}]}\end{array}$ \\
\hline 18 & 23 & 100 & 0 \\
14 & 23 & 50 & 0 \\
33 & 23 & 50 & -6.27 \\
15 & 60 & 50 & -6.27 \\
14 & 23 & 50 & -6.27 \\
$7^{*}$ & 60 & 50 & -6.27 \\
$7^{*}$ & 23 & 50 & -6.27 \\
7 & 60 & 50 & -6.27 \\
13 & 23 & 50 & -6.27 \\
14 & 23 & 50 & 0 \\
\hline
\end{tabular}

The four parameters of the B3 model describing the basic creep, $q_{1}, q_{2}, q_{3}$ and $q_{4}$, were determined from the composition of the concrete mixture and from the compressive strength using empirical formulae according to [1]. The result of this prediction exceeded expectations; only minor adjustments were necessary to get the optimal fit (see the first part of the strain evolution in Figure 6). The following values were used: $q_{1}=19.5, q_{2}=160, q_{3}=5.25$ and $q_{4}=12.5$ (all in $10^{-6} / \mathrm{MPa}$ ). They differ significantly from the values recommended in [2], $q_{1}=25$, $q_{2}=100, q_{3}=1.5$ and $q_{4}=6$, which do not provide satisfactory agreement with experimental data.

MPS theory uses three additional parameters, $c_{0}$, $k_{1}$ and $c$, but parameter $c$ should be equal to $c_{0} q_{4}$. It has been found that the remaining parameters $c_{0}$ and $k_{1}$ are not independent. What matters for creep is only their product. For different combinations of $c_{0}$ and $k_{1}$ giving the same product, the evolution of microprestress is different but the evolution of creep strain is exactly the same. Since microprestress is not directly measurable, $c_{0}$ and $k_{1}$ cannot (and need not) be determined separately. In practical computations, $k_{1}$ can be set to a fixed value (eg. $1 \mathrm{MPa} / \mathrm{K}$ ), and $c_{0}$ can be varied until the best fit with experimental data is obtained; in all the following figures $c_{0}$ is specified in $\mathrm{MPa}^{-1}$ day $^{-1}$. All other parameters were used according to standard recommendations.

A really good fit of the first experimental data set $(98 \%$ relative humidity, i.e., $h=0.98)$ was obtained for $c_{0}=0.235 \mathrm{MPa}^{-1} \mathrm{day}^{-1}$; see Figure 6 . The agreement is satisfactory except for the last interval, which corresponds to unloading. It is worth noting that the thermally induced part of creep accounts for more than a half of the total creep (compare the experimental data with the solid curve labeled basic in Figure 6). Unfortunately, with default values of the other parameters, the same value of $c_{0}$ could not be used to fit experimental data set number 2 , because it would have led to overestimation of the creep (see the dashed curve in Figure 7). In the first loading interval of 37 days, creep takes place at room temperature and the best agreement would be obtained with parameter $c_{0}$ set to $0.940 \mathrm{MPa}^{-1} \mathrm{day}^{-1}$; see the dashdotted curve in Figure 7. However, at the later stage when the temperature rises to $60^{\circ} \mathrm{C}$, the creep would be grossly overestimated. A reasonable agreement during this stage of loading is obtained with $c_{0}$ reduced to $0.067 \mathrm{MPa}^{-1}$ day $^{-1}$ (solid curve in Figure 7), but then the creep is underestimated in the first interval in Figure 6 left. Raising parameter $\alpha_{S}$ from its recommended value 0.1 to 0.3 (short-dashed curve in Figure 7 right) has approximately the same effect as decreasing $c_{0}$ from 0.235 to $0.067 \mathrm{MPa}^{-1} \mathrm{day}^{-1}$. Parameter $\alpha_{S}$ controls the effect of reduced humidity on the rate of microprestress relaxation, and its modification has no effect on the response of sealed specimens.

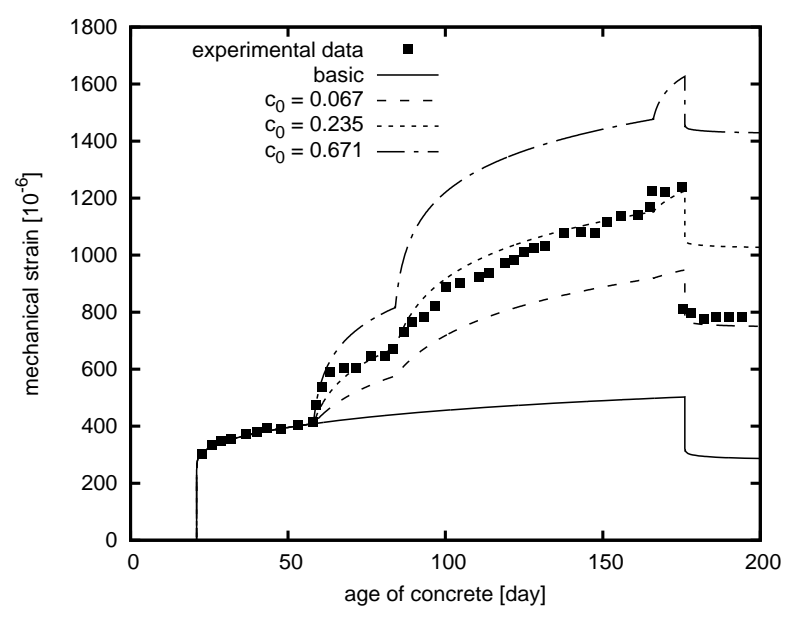

Fig. 6: Mechanical strain evolution for sealed specimens, with relative pore humidity assumed to be $98 \%$, loaded by compressive stress $6.27 \mathrm{MPa}$ at time $t^{\prime}=21$ days

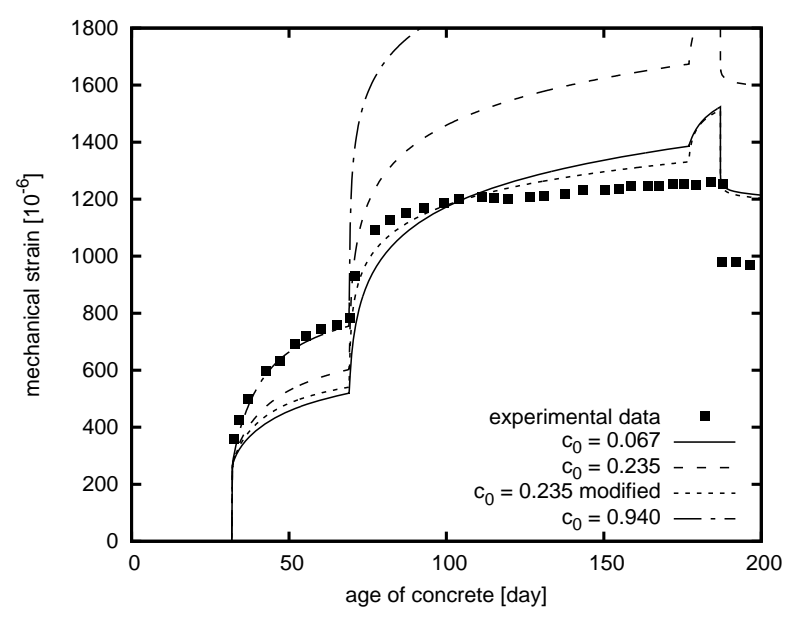

Fig. 7: Mechanical strain evolution for drying specimens at $50 \%$ relative environmental humidity, loaded by compressive stress $6.27 \mathrm{MPa}$ at time $t^{\prime}=32$ days 


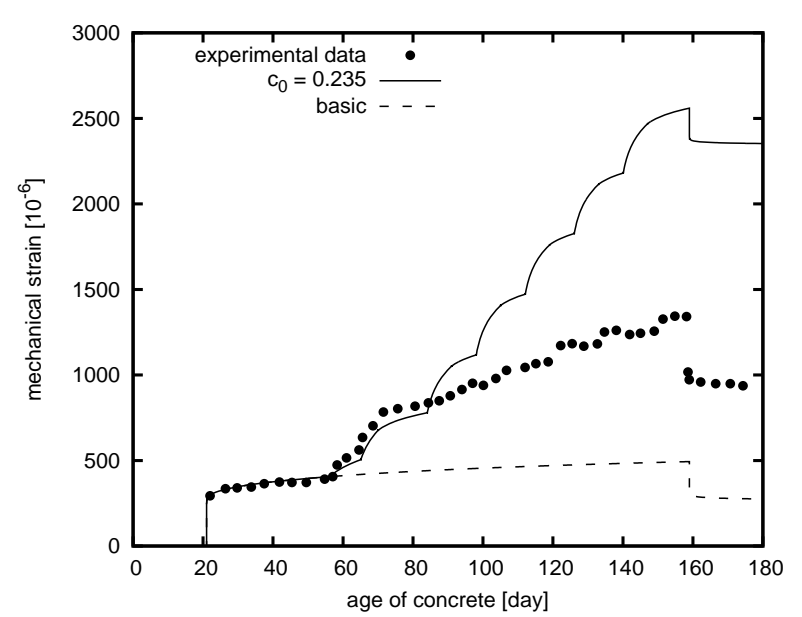

Fig. 8: Mechanical strain evolution for sealed specimen, loaded by compressive stress $6.27 \mathrm{MPa}$ at time $t^{\prime}=21$ days and subjected to cyclic variations of temperature

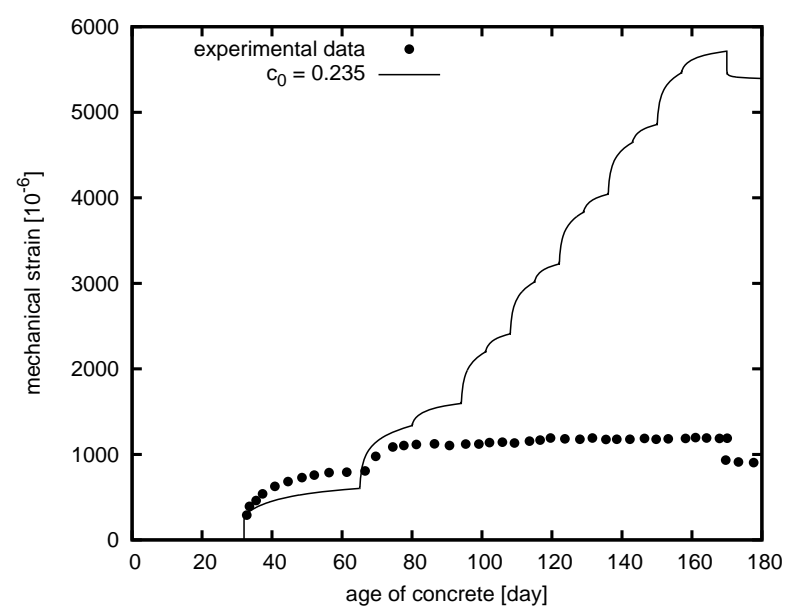

Fig. 9: Mechanical strain evolution for drying specimens, loaded by compressive stress $6.27 \mathrm{MPa}$ at time $t^{\prime}=32$ days and subjected to cyclic variations of temperature

For the last two testing programs described in Tables 3 and 4, the agreement between the experimental and computed data is reasonable only until the end of the second heating cycle (solid curves in Figure 8 and Figure 9). For data set 3, the final predicted compliance exceeds the measured value almost twice (Figure 8), for data set 4 almost five times (Figure 9). In order to obtain a better agreement, parameter $c_{0}$ would have to be reduced, but this would result in an underestimation of the creep in the first two testing programs. The experimental data show that the temperature cycles significantly increase the creep only in the first cycle; during subsequent thermal cycling their effect on creep diminishes. Therefore it could be beneficial to enhance the material model by adding internal memory, which would improve the behavior under cyclic thermal loading, while the response to sustained loading would remain unchanged.
Another deficiency of the model is illustrated by the graphs in Figure 10. They refer to the first set of experiments. As documented by the solid curve in Figure 6, a good fit was obtained by setting parameter $c_{0}=0.235 \mathrm{MPa}^{-1} \mathrm{day}^{-1}$, assuming that the relative pore humidity is $98 \%$. The pores are initially completely filled with water; however, even if the specimen is perfectly sealed, the relative humidity decreases slightly due to the water deficiency caused by the hydration reaction. This phenomenon is referred to as self-desiccation.

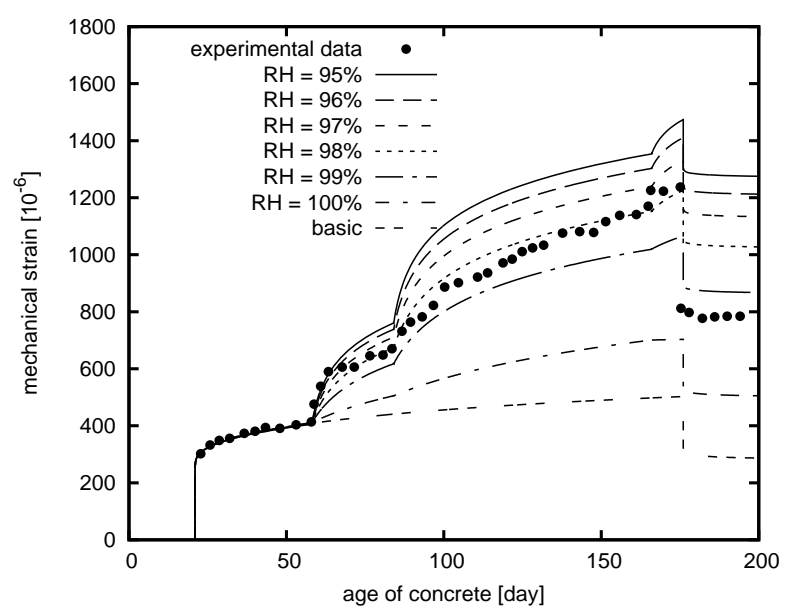

Fig. 10: Mechanical strain evolution for sealed specimens, loaded by compressive stress $6.27 \mathrm{MPa}$ from age 21 days, with the assumed relative humidity of the pores varied from $95 \%$ to $100 \%$. Parameters of MPS theory: $k_{1}=1 \mathrm{MPa} / \mathrm{K}, c_{0}=0.235 \mathrm{MPa}^{-1}$ day $^{-1}$

The problem is that the exact value of pore humidity in a sealed specimen and its evolution in time are difficult to determine. In simple engineering calculations, a constant value of $98 \%$ is often used. Unfortunately, the response of the model is quite sensitive to this choice, and the creep curves obtained with other assumed values of pore humidity in the range from $95 \%$ to $100 \%$ would be different; see Figure 10 . The source this strong sensitivity is the assumption that the instantaneously generated microprestress is proportional to the absolute value of the change of $T \ln (h)$; see the right-hand side of (2). Rewriting (2) as

$$
\frac{\mathrm{d} S}{\mathrm{~d} t}+\psi_{S}(T, h) c_{0} S^{2}=k_{1}\left|\ln h \frac{\mathrm{d} T}{\mathrm{~d} t}+\frac{T}{h} \frac{\mathrm{d} h}{\mathrm{~d} t}\right|
$$

we can see that at (almost) constant humidity close to $100 \%$, the right-hand side is proportional to the magnitude of the temperature rate, with proportionality factor $k_{1}|\ln (h)| \approx k_{1}(1-h)$. For instance, if the assumed humidity is changed from $99 \%$ to $98 \%$, this proportionality factor is doubled. 


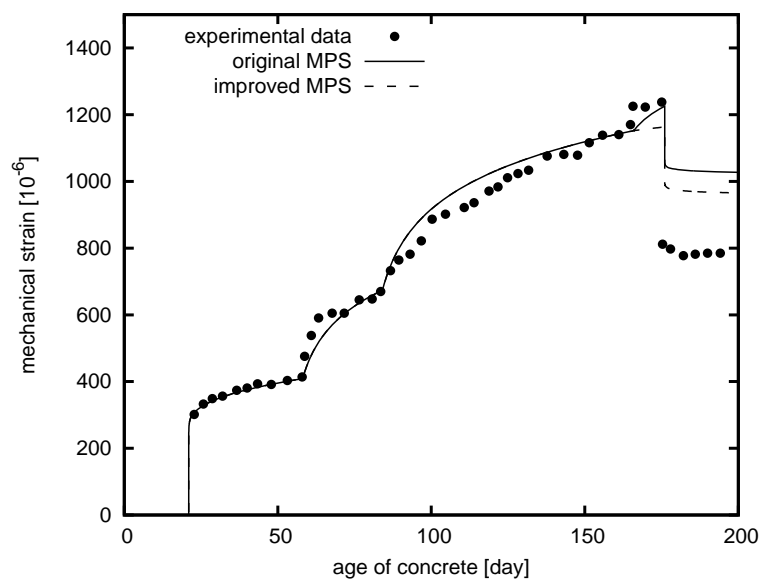

Fig. 11: Mechanical strain evolution for sealed specimens loaded by compressive stress $6.27 \mathrm{MPa}$ at time $t^{\prime}=21$ days

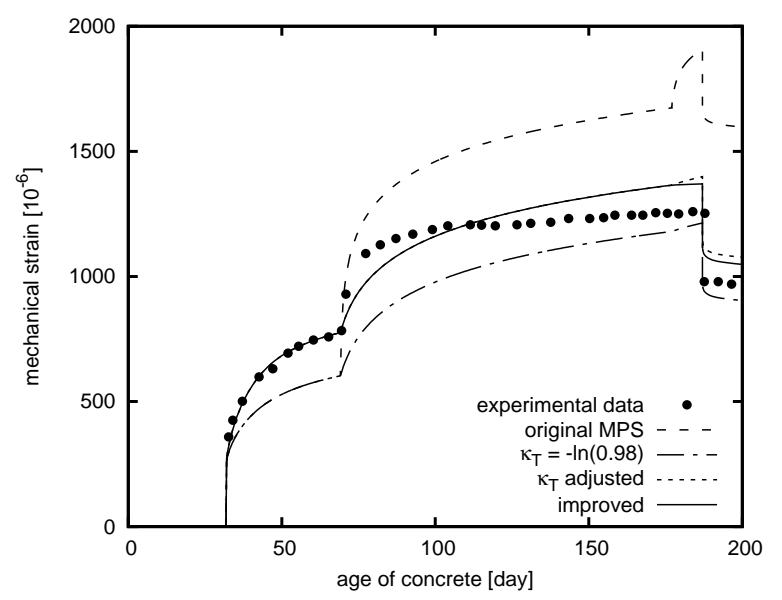

Fig. 12: Mechanical strain evolution for drying specimens loaded by compressive stress $6.27 \mathrm{MPa}$ at time $t^{\prime}=32$ days

\section{Improved material model and its validation}

As a simple remedy to overcome these problems, the microprestress relaxation equation (2) is replaced by

$$
\begin{aligned}
\frac{\mathrm{d} S}{\mathrm{~d} t}+\psi_{S}(T, h) c_{0} S^{2} & =k_{1}\left|\frac{T}{h} \frac{\mathrm{d} h}{\mathrm{~d} t}-\kappa_{T} k_{T}(T) \frac{\mathrm{d} T}{\mathrm{~d} t}\right| \\
\text { with } \quad k_{T}(T) & =\mathrm{e}^{-c_{T}\left(T_{\max }-T\right)}
\end{aligned}
$$

in which $\kappa_{T}$ and $c_{T}$ are new parameters and $T_{\max }$ is the maximum reached temperature. With $\kappa_{T}=0.02$, the creep curves in Figure 10 plotted for different assumed pore humidities would be almost identical with the solid curve, which nicely fits the experimental results. The introduction of a new parameter provides more flexibility, which is needed to improve the fit of the second testing program in Figure 7, with combined effects of drying and temperature varia- tion. For sealed specimens and monotonous thermal loading, only the product $c_{0} k_{1} \kappa_{T}$ matters, and so the good fit in Figure 7 could be obtained with different combinations of $\kappa_{T}$ and $c_{0}$.

The results are shown in Figures 11 and 12 for sustained thermal loading (data sets 1 and 2) and in Figures 13 and 14 for cyclic thermal loading (data sets 3 and 4). Default values of parameters $\alpha_{S}, \alpha_{R}, \alpha_{E}$ and activation energies are used. In these plots, data series labeled original MPS show results obtained with standard MPS.

The data series $\kappa_{T}=-\ln (0.98)$ were obtained with $c_{0}=0.235 \mathrm{MPa}^{-1}$ day $^{-1}, k_{1}=1 \mathrm{MPa} / \mathrm{K}, \kappa_{T}=$ 0.020203 and $c_{T}=0$. The data series $\kappa_{T}$ adjusted correspond to parameters $c_{0}=0.235 \mathrm{MPa}^{-1} \mathrm{day}^{-1}$, $k_{1}=4 \mathrm{MPa} / \mathrm{K}, \kappa_{T}=0.005051$ and $c_{T}=0$. Note that in the case of constant relative humidity (Figures 11 and 13) these series coincide with the data series original MPS.

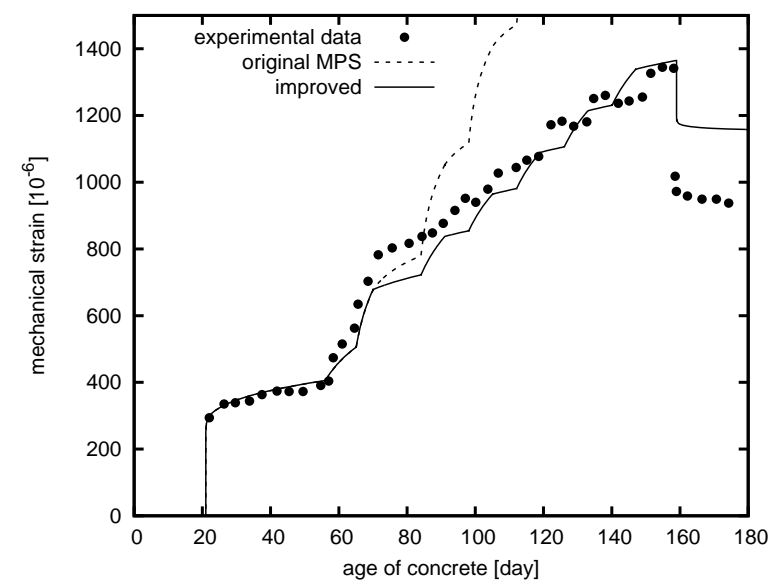

Fig. 13: Mechanical strain evolution for sealed specimen, loaded by compressive stress $6.27 \mathrm{MPa}$ at time $t^{\prime}=21$ days and subjected to cyclic variations of temperature

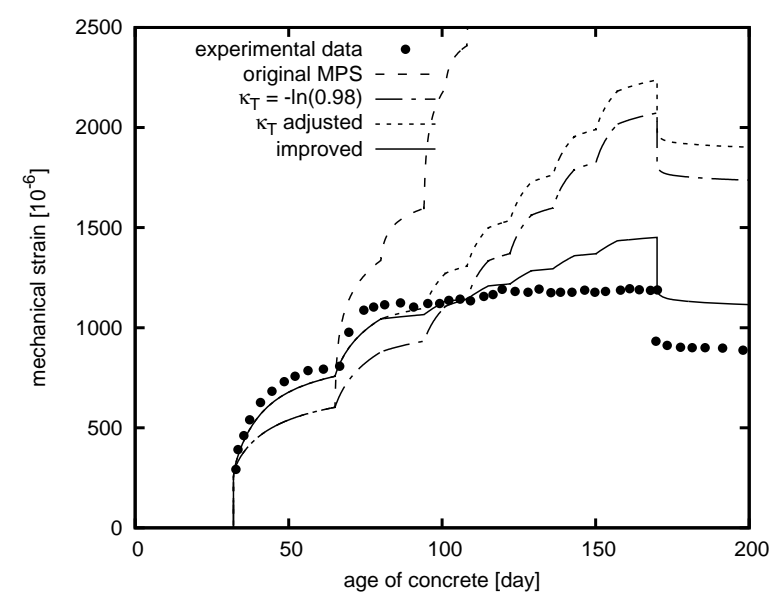

Fig. 14: Mechanical strain evolution for drying specimens, loaded by compressive stress $6.27 \mathrm{MPa}$ at time $t^{\prime}=32$ days and subjected to cyclic variations of temperature 
The best agreement with experimental data is obtained with $c_{0}=0.235 \mathrm{MPa}^{-1}$ day $^{-1}, k_{1}=4 \mathrm{MPa} / \mathrm{K}$, $\kappa_{T}=0.005051$ and $c_{T}=0.3 \mathrm{~K}^{-1}$; these series are labeled improved. In Figure 11, only a small change can be observed compared to data series original $M P S$; these differences arise when the temperature ceases to be monotonous. For the sealed specimen (Figure 11), this change is detrimental, but looking at Figures 13 and 14, this deterioration is negligible compared to the substantial improvement in the case of cyclic thermal loading.

\section{Conclusions}

The material model based on MPS theory has been successfully implemented into the OOFEM finite element package, and has been used in simulations of concrete creep at variable temperature and humidity.

MPS theory performs well for standard sustained levels of temperature and load levels within the linear range of creep, provided that the activation energy is properly adjusted. For higher sustained temperatures (above $70^{\circ} \mathrm{C}$ ) the experimental data are reproduced with somewhat lower accuracy.

For sealed specimens subjected to variable temperature, the results predicted by MPS theory are very sensitive to the assumed value of relative pore humidity (which is slightly below $100 \%$ due to selfdesiccation). In order to overcome this deficiency, a modified version of the model has been proposed and successfully validated. Excessive sensitivity to the specific choice of relative humidity has been eliminated. Also, it has become easier to calibrate the model because thermal and moisture effects on creep are partially separated.

The original MPS theory grossly overestimates creep when the specimen is subjected to cyclic temperature. A new variable $k_{T}$ has been introduced in order to reduce the influence of subsequent thermal cycles on creep. This modification does not affect creep tests in which the evolution of temperature is monotonous.

\section{Acknowledgement}

Financial support for this work was provided by projects $103 / 09 / \mathrm{H} 078$ and $\mathrm{P} 105 / 10 / 2400$ of the Czech Science Foundation. The financial support is gratefully acknowledged.

\section{References}

[1] Bažant, Z. P., Baweja, S.: Creep and shrinkage prediction model for analysis and design of concrete structures: Model B3, Adam Neville Symposium: Creep and Shrinkage - Structural Design Effects, 2000.
[2] Bažant, Z. P., Cedolin, L., Cusatis, G.: Temperature effect on concrete creep modeled by microprestress-solidification theory, Journal of Engineering Mechanics, 2004, 130, 6, 691-699.

[3] Bažant, Z. P., Hauggaard, A. B., Ulm, F.: Microprestress-solidification theory for concrete creep. I: Aging and drying effects, Journal of Engineering Mechanics, 1997, 123, 11, 1188-1 194.

[4] Bažant, Z. P., Hauggaard, A. B., Ulm, F.: Microprestress-solidification theory for concrete creep. II: Algorithm and verification, Journal of Engineering Mechanics, 1997, 123, 11, 1 195-1201.

[5] Bažant, Z. P., Prasannan, S.: Solidification theory for concrete creep. I: Formulation, Journal of Engineering Mechanics, 1989, 115, 8, 1691-1 703 .

[6] Bažant, Z. P., Prasannan, S.: Solidification theory for concrete creep. II: Verification and Application, Journal of Engineering Mechanics, 1989, 115, 8, 1704-1725.

[7] Fahmi, H. M., Polivka, M., Bresler, B.: Effects of sustained and cyclic elevated temperature on creep of concrete, Cement and Concrete Research, 1972, 2, 591-606.

[8] Kommendant, G. J., Polivka, M., Pirtz, D.: Study of concrete properties for prestressed concrete reactor vessels, final report - part II, Creep and strength characteristics of concrete at elevated temperatures, Rep. no. UCSESM 76-3 prepared for General Atomic Company, Berkeley : Dept. Civil Engineering, Univ. of California, 1976.

[9] Nasser, K. W., Neville, A. M.: Creep of concrete at elevated temperatures, ACI Journal, 1965, 62, $1567-1579$.

[10] Patzák, B.: OOFEM home page, http://www.oofem.org, 2000.

[11] Patzák, B., Bittnar, Z.: Design of object oriented finite element code, Advances in Engineering Software, 2001, 32, 10-11, 759-767.

[12] Patzák, B., Rypl, D., Bittnar, Z.: Parallel explicit finite element dynamics with nonlocal constitutive models, Computers $\&$ Structures, 2001, 79, 26-28, 2 287-2 297.

Petr Havlásek

E-mail: petr.havlasek@fsv.cvut.cz

Milan Jirásek

E-mail: milan.jirasek@fsv.cvut.cz

Department of Mechanics

Faculty of Civil Engineering

Czech Technical University in Prague

Thákurova 7, 16629 Prague 6 